\begin{tabular}{|c|c|}
\hline Title & $\begin{array}{l}\text { Melatonin suppression during a simulated night shift in medium intensity light is increased by } 10 \text {-minute breaks in dim } \\
\text { light and decreased by } 10 \text {-minute breaks in bright light }\end{array}$ \\
\hline Author(s) & $\begin{array}{l}\text { Lee, Sang-II; Kinoshita, Saki; Noguchi, Anna; Eto, Taisuke; Ohashi, Michihiro; Nishimura, Y uki; Maeda, Kaho; } \\
\text { Motomura, Y uki; A wata, Y asuhiro; Higuchi, Shigekazu }\end{array}$ \\
\hline Citation & $\begin{array}{l}\text { Chronobiology International, 37(6), 897-909 } \\
\text { https://doi.org/10.1080/07420528.2020.1752704 }\end{array}$ \\
\hline Issue Date & $2020-06$ \\
\hline Doc URL & http:/hdl .handle.net/2115/81619 \\
\hline Rights & $\begin{array}{l}\text { This is an A ccepted Manuscript of an article published by Taylor \& Francis in Chronobiology International on June } \\
\text { 2020, avail able online: http:/www.tandfonline.com/10.1080/07420528.2020.1752704. }\end{array}$ \\
\hline Type & article (author version) \\
\hline File Information & LCBI-2019-0234.R2_Proof_hi2.pdf \\
\hline
\end{tabular}

Instructions for use 


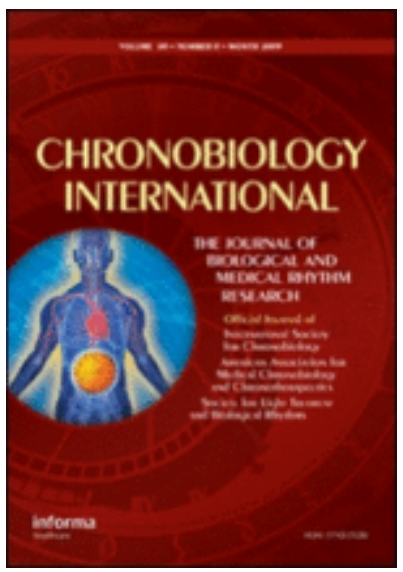

\section{Melatonin suppression during a simulated night shift in medium intensity light is increased by 10 -minute breaks in dim light and decreased by 10-minute breaks in bright light}

\begin{tabular}{|c|c|}
\hline Journal: & Chronobiology International \\
\hline Manuscript ID & LCBI-2019-0234.R2 \\
\hline Manuscript Type: & Original Reports \\
\hline $\begin{array}{r}\text { Date Submitted by the } \\
\text { Author: }\end{array}$ & $n / a$ \\
\hline Complete List of Authors: & $\begin{array}{l}\text { LEE, SANG-IL; Kyushu University Graduate School of Design Faculty of } \\
\text { Design, Department of Human Science; Hokkaido University Faculty of } \\
\text { Engineering, Division on Human Environmental Systems } \\
\text { Kinoshita, Saki; Kyushu University, Graduate School of Integrated } \\
\text { Frontier Science, Department of Kansei Science } \\
\text { Noguchi, Anna; Kyushu University, Graduate School of Integrated } \\
\text { Frontier Science, Department of Kansei Science } \\
\text { Eto, Taisuke; Kyushu University, Graduate School of Integrated Frontier } \\
\text { Science, Department of Kansei Science } \\
\text { Ohashi, Michihiro; Kyushu University, Graduate School of Integrated } \\
\text { Frontier Science, Department of Kansei Science Email address } \\
\text { Nishimura, Yuki; Kyushu University, Graduate School of Integrated } \\
\text { Frontier Science, Department of Kansei Science Email address; National } \\
\text { Institute of Occupational Safety and Health Japan Noborito District } \\
\text { Maeda, Kaho; Japan Aerospace Exploration Agency Institute of } \\
\text { Aerospace Technology Tsukuba Space Center, Ground Facilities } \\
\text { Department } \\
\text { Motomura, Yuki; Kyushu University Graduate School of Design Faculty of } \\
\text { Design, Department of Human Science } \\
\text { Awata, Yasuhiro; Japan Aerospace Exploration Agency Institute of } \\
\text { Aerospace Technology Tsukuba Space Center, Ground Facilities } \\
\text { Department } \\
\text { Higuchi, Shigekazu; Kyushu University Graduate School of Design } \\
\text { Faculty of Design, Department of Human Science }\end{array}$ \\
\hline Keywords: & $\begin{array}{l}\text { Humans, Night shift work, Intermittent light at night exposure, Light } \\
\text { adaptation, Short duration, Melatonin suppression, Subjective } \\
\text { sleepiness, Performance }\end{array}$ \\
\hline
\end{tabular}


1

2

3

4

5

6

7

8

9

10

11

12

13

14

15

16

17

18

19

20

21

22

23

24

25

26

27

28

29

30

31

32

33

34

35

36

37

38

39

40

41

42

43

44

45

46

47

48

49

50

51

52

53

54

55

56

57

58

59

60

\section{SCHOLARONE ${ }^{\text {W }}$ \\ Manuscripts}

URL: http://mc.manuscriptcentral.com/lcbi E-mail: ICBI-peerreview@journals.tandf.co.uk 


\section{Melatonin suppression during a simulated night shift in medium intensity light is increased by 10-minute breaks in dim light and decreased by 10-minute breaks in bright light}

Sang-il Lee ${ }^{\mathrm{a}, \mathrm{b}}$, Saki Kinoshita ${ }^{\mathrm{c}}$, Anna Noguchic, Taisuke Eto ${ }^{\mathrm{c}}$, Michihiro

Ohashic $^{c}$, Yuki Nishimura ${ }^{\mathrm{c}, \mathrm{e}}$, Kaho Maeda ${ }^{\mathrm{d}}$, Yuki Motomura ${ }^{\mathrm{a}}$, Yasuhiro

Awata $^{\mathrm{d}}$, and Shigekazu Higuchi* ${ }^{*}$

${ }^{a}$ Department of Human Science, Faculty of Design, Kyushu University, Fukuoka, Japan; ${ }^{b}$ Division of Human Environmental Systems, Faculty of Engineering, Hokkaido University, Sapporo, Japan; 'Department of Kansei Science, Graduate School of Integrated Frontier Science, Kyushu University, Fukuoka, Japan; ${ }^{d}$ Ground Facilities Department, Japan Aerospace Exploration Agency, Tsukuba, Japan; ${ }^{e}$ National Institute of Occupational Safety and Health, Kawasaki, Japan

*corresponding author:

Shigekazu Higuchi, Ph.D

Department of Human Science

Faculty of design, Kyushu University

4-9-1 Shiobaru, Minami-ku, Fukuoka, 815-8540, Japan 


\section{Abstract}

Exposure to light at night results in disruption of endogenous circadian rhythmicity and/or suppression of pineal melatonin, which can consequently lead to acute or chronic adverse health problems. In the present study, we investigated whether exposure to very dim light or very bright light for a short duration influences melatonin suppression, subjective sleepiness, and performance during exposure to constant moderately bright light. Twenty-four healthy male university students were divided into two experimental groups: Half of them (mean age: $20.0 \pm 0.9$ years) participated in an experiment for short-duration (10 min) light conditions of medium intensity light (430 lx, medium breaks) vs. very $\operatorname{dim} \operatorname{light}(<1 \mathrm{~lx}, \operatorname{dim}$ breaks) and the other half (mean age: $21.3 \pm 2.5$ years) participated in an experiment for short-duration light conditions of medium intensity light (430 lx, medium breaks) vs. very bright light (4700 1x, bright breaks). Each simulated night shift consisting of 5 sets (each including 50-minute night work and 10-minute break) was performed from 01:00 to 06:00h. The subjects were exposed to medium intensity light (550 lx) during the night work. Each 10-minute break was conducted every hour from 02:00 to 06:00h. Salivary melatonin concentrations were measured, subjective sleepiness was assessed, the psychomotor vigilance task was performed at hourly intervals from 21:00h until the end of the experiment. Compared to melatonin suppression between 04:00 and $06: 00 \mathrm{~h}$ in the condition of medium breaks, the condition of dim breaks significantly promoted melatonin suppression and the condition of bright breaks significantly diminished melatonin suppression. However, there was no remarkable effect of either dim breaks or bright breaks on subjective sleepiness and performance of the psychomotor vigilance task. Our findings suggest that periodic exposure to light for short durations during exposure to a constant light environment affects the sensitivity of pineal melatonin to constant light depending on the difference between light intensities in the two light conditions (i.e., short light exposure vs. constant light exposure). Also, our findings indicate that exposure to light of various intensities at night could be a factor influencing the light-induced melatonin suppression in real night work settings.

Keywords: Humans; Night shift work; Intermittent light at night exposure; Light adaptation; Short duration; Melatonin suppression; Subjective sleepiness; Performance 


\section{Introduction}

The 24-h light-dark cycle in nature is known as the strongest zeitgeber (i.e., time giver) for almost all mammalians. In humans, the suprachiasmatic nuclei (SCN), i.e., the circadian pacemaker, regulates circadian rhythmicity throughout the body via phototransduction input from ganglion cells in the retina (Weaver 1998). For instance, the SCN restrains pineal melatonin production during the daytime and allows melatonin secretion during the night. It has been believed that the release timing and the amount of melatonin secretion are related to the regulation of physiological and behavioral circadian rhythm (Macchi \& Bruce 2004). However, the extension of daylight duration in modern life by using artificial lighting at night is likely to lead to acute melatonin suppression and circadian disturbances, which are partially responsible for some health problems (Smolensky et al. 2015; Smolensky et al. 2016; Lunn et al. 2017; Touitou et al. 2017).

The magnitude of melatonin suppression varies depending on light intensity (Zeitzer et al. 2000), exposure duration (Aoki et al. 1998), and wavelength composition (Brainard et al. 2001). Recently, however, there is growing evidence that prior light history has an impact on the magnitude of melatonin suppression in response to a subsequent light stimulus at night. Several studies have suggested that less daylight can increase melatonin sensitivity to light at night (Hebert et al. 2002; Smith et al. 2004). A field study with human subjects showed that melatonin suppression in response to 500 lx light at night was greater following exposure to dim light (wearing dark goggles with $2 \%$ transmission lenses) for one week than following exposure to bright light for one week (Hebert et al. 2002). Similar results were obtained in laboratory studies on melatonin suppression (Smith et al. 2004) and circadian phase shift (Chang et al. 2011). The results of those studies, however, were likely to have been affected by great differences in the intensities of prior light conditions. One study, however, showed significantly dampened melatonin suppression in response to blue light $(460 \mathrm{~nm}$ monochromatic light) following 2-h exposure to dim white light (18 lx) compared to that following 2-h dark adaptation (Jasser et al. 2006). Taken together, the results suggest that melatonin sensitivity to a light stimulus can be increased or decreased depending on the relative intensity of a prior light stimulus to the target light stimulus.

Although the mechanism involved in the effect of prior light history is not clear, it seems that a long period of photic adaptation alters the absolute response threshold of photoreceptors and/or photosensory inputs from the photoreceptors to the 
69 SCN. In humans, the visual photoreceptors (i.e., rods and cones) and especially a small 70 subset of retinal ganglion cells expressing melanopsin (mRGC) contribute to non-visual 71 effects on the circadian system (Gooley et al. 2012); those are designed to adapt to various changes in ambient light in a short time. For instance, exposure of the eyes to bright light desensitizes the visual photoreceptors to facilitate response to intensity increment (i.e., light adaptation) (Fain et al. 2001). Conversely, darkness fully recovers the photoreceptors from the desensitized state (i.e., dark adaptation) (Lamb \& Pugh 2004). It should be noted, however, that the time required for photo-regeneration becomes longer as the photopigment bleaches more. Recently, evidence of the adaptation capacity of mRGC to light has been provided (Wong et al. 2005). One study with young human subjects, for example, showed that blocking short-wavelength light, which dramatically activates the mRGC, by using orange-colored contact lenses (i.e., blue light-filtering lenses) immediately reduced melatonin suppression but that the reduction in melatonin suppression disappeared 16 days after wearing the blue lightfiltering lenses (Gimenez et al. 2014).

If two lights of different intensities are emitted alternately for long or short durations, can the effects of the short duration exposure be ignored? Some researchers have demonstrated that using intermittent light (e.g., alternate exposure to bright light and darkness) during the night for multiple days is effective for shifting the phase of human circadian rhythm (Baehr et al. 1999; Rimmer et al. 2000; Crowley et al. 2003; Gronfier et al. 2004; Smith et al. 2009). A mathematical model of the effects of brief light on the human circadian pacemaker has been proposed for explaining the results of previous investigations (Kronauer et al. 1999). Recent studies, however, showed that even brief (12 minutes or shorter) exposure to bright light was able to elicit circadian phase delay and melatonin suppression after previous adaptation to dim light during a constant routine (Chang et al. 2012; Rahman et al. 2017).

Although there is a possibility that exposure to darkness or dimmer light or brighter light can alter (at least temporarily) the sensitivity of non-visual responses to a subsequent light stimulus, the intermittent light conditions used in most previous studies are far from real working conditions. Furthermore, the effects of short exposure to very dim light during night work under light of constant medium intensity are unknown. In

100 the present study, we therefore investigated whether short-duration exposure to very dim light or very bright light influences melatonin suppression, subjective sleepiness,

102 and performance during exposure to constant light of medium intensity. 
Materials and Methods

105

\section{Subjects}

106 Twenty-four healthy male university students participated in this study. Twelve subjects

107 (mean \pm SD age: $20.0 \pm 0.9$ years) participated in Experiment 1 and the remaining 12

108 subjects (mean \pm SD age: $21.3 \pm 2.5$ years) participated in Experiment 2. None of the

109 participants showed extreme morningness or extreme eveningness as assessed by a

110 Japanese version of the Morningness-Eveningness Questionnaire (Ishihara et al. 1984).

111 Subjects who had engaged in night shift work or who had experienced time zone travel

112 (i.e., at least $>1$ time zone) in the previous three months were excluded from the study.

113 Signed written informed consent to take part in the research study, which was approved

114 by the Ethical Committee of Kyushu University, was obtained from all participants. The

115 experiments were conducted in accordance with the Declaration of Helsinki.

116

\section{Experimental light conditions}

118 The vertical illuminance and irradiance of each light condition were measured at eye

119 level in a sitting position using an illuminance spectroradiometer (CL-500A, KONICA

120 MINOLTA INC., Japan). Two experimental chambers were used for the experiment

121 (chamber 1) and for 10 min breaks (chamber 2). In chamber 1, light-emitting diode

122 (LED) ceiling lights (HH-LC569A, Panasonic Inc., Japan) were set up for exposure to

123 constant medium intensity light ( 550 lx). Fluorescent ceiling lights (FPL36CW, Panasonic

124 Inc., Japan) were used for medium breaks ( $430 \mathrm{~lx})$ or bright breaks ( $4700 \mathrm{~lx})$. For dim

125 breaks $(<1 \mathrm{~lx})$, incandescent bulbs were installed on the floor as indirect lighting.

126 Detailed information on each light condition is given in Table 1. Melanopic lux was

127 calculated using an excel-based toolbox provided by the Lucas Group at the University

128 of Manchester (Enezi et al. 2011).

129 (http://lucasgroup.lab.manchester.ac.uk/measuringmelanopicilluminance/).

\section{$131 \quad$ Procedure}

132 Two experiments with a crossover design, including dim breaks vs. medium breaks

133 (Experiment 1) and bright breaks vs. medium breaks (Experiment 2), were conducted to

134 investigate the effects of each short-duration (10 $\mathrm{min})$ light exposure condition on

135 melatonin suppression, subjective sleepiness, and performance during exposure to

136 constant bright light. Each participant was therefore required to visit our laboratory 
137 twice with an interval of 2 weeks. Six participants simultaneously participated in each 138 experiment. Prior to the experiment, participants were instructed to sleep for more than

1397 hours between 00:00 and 08:00h for one week. An accelerometry-based activity 140 monitor (Lifecorder plus, Suzuken Co Ltd, Japan) and daily sleep diary were used to 141 confirm the implementation of sleep intervention during the control period.

142 Furthermore, each participant sent us a message via a mobile phone shortly before 143 bedtime and shortly after waking up. If there was no message from a participant, we 144 called the participant to confirm his situation. The participants were instructed not to 145 drink alcohol from three days before the experiment. Also, excessive exercise, napping, 146 and caffeine consumption were not allowed commencing the day before the experiment. 147 The procedures used for Experiment 1 and Experiment 2 were same (Figure 1). 148 The participants arrived at the experimental facility at about 12:00h and dressed into the 149 experimental clothes (short-sleeved T-shirt, short pants, and no socks) after receiving 150 brief instructions for the experiment. The participants stayed in chamber 1 in a sitting 151 position from 13:00h until the end of the experiment. The room illuminance was $275 \mathrm{~lx}$ 152 (vertical illuminance at eye level) from 13:00 to 19:00h. The light illuminance was then 153 changed into a dim light $(<10 \mathrm{~lx})$ from 19:00 to 01:00h. The participants were allowed 154 to use portable devices (e.g., smartphone, tablet pc, laptop, set to minimum brightness) 155 and read books between 19:00 and 00:00h. For reference, the illuminances of the self156 illuminating portable devices were measured under $2 \mathrm{~lx}$ at a distance of $20 \mathrm{~cm}$ from the 157 center of each screen. The participants started simulated night work in a sitting position 158 from 00:00 to 06:00h (practice session between 00:00 and 01:00h) in chamber 1 . The 159 light illuminance was changed to medium intensity light (550 lx) from 01:00 until 06:00h. 160 Each break condition was conducted every hour from 02:00 to 06:00h in chamber 2 and 161 lasted $10 \mathrm{~min}$. During each break, all of the participants performed light stretching $(\sim 2$ $162 \mathrm{~min}$ in a standing position) and a word chain game (Shiritori, $\sim 5 \mathrm{~min}$ in a sitting 163 position) and answered questionnaires ( $\sim 3 \mathrm{~min}$ in a sitting position) that addressed 164 subjective sleepiness (Karolinska sleepiness scale, KSS) and 'dummy variables' (e.g., 165 physical fatigue and mood state). Each break was followed by 50-min of simulated 166 night work in which the participants conducted PVT and answered the KSS and dummy 167 questionnaires for $10 \mathrm{~min}$ followed by card games for $20 \mathrm{~min}$. After a 5-min rest period, 168 the participants conducted PVT, collected saliva, and answered the KSS for $15 \mathrm{~min}$ 169 (Figure 1b). The order of the break conditions in each experiment (i.e., Experiment 1 or 170 Experiment 2) was random for each participant. For example, some participants 
171 performed the medium breaks on the first visit and the bright breaks (or dim breaks) on

172 the next visit, and vice versa for other participants. Participants had dinner at 19:00h

173 (typical Japanese food, the same dishes for all participants in every experiment) and a

174 late-night snack at 23:30h (rice, miso soup). No drinks except for water were provided

175 throughout each experiment.

176 Salivary samples were collected hourly using a plain cotton plug (Salivette

177 Sarstedt, Germany) from 21:00h until the end of the experiment. Participants did not

178 drink any water for 15 min prior to each salivary sample collection. Subjective

179 sleepiness was evaluated using the KSS at 1-hour intervals from 21:00 to 06:00h, $5 \mathrm{~min}$

180 before collecting the salivary sample. Subjective sleepiness during each break was also

181 evaluated to confirm the acute effects of the break on sleepiness. Each participant

182 performed the Psychomotor Vigilance Task (PVT) twice with a 1-hour interval, soon

183 after the break and shortly before the next break. The PVT was performed for 5 min

184 using Presentation (Neurobehavioral Systems Inc., Albany, CA). A visual stimulus was

185 displayed randomly on the PC display at intervals of 2 to $10 \mathrm{sec}$. Participants were

186 instructed to press the space key on the keyboard as soon as possible after the

187 appearance of the visual stimulus. A beep sound was emitted from each earphone if the

188 participants did not react within $3000 \mathrm{msec}$.

\section{Sample analysis}

191 Salivary melatonin concentrations were measured by radioimmunoassay kit (RK-DSM;

192 Buhlmann Laboratories AG, Allschwil, Switzerland). Melatonin area under the curve

193 (AUC; trapezoidal approximation) between 21:00 and 06:00h was calculated to evaluate

194 the overall effect of each break condition on melatonin suppression. Data of three

195 participants were excluded from analysis of the results for bright breaks, since each

196 participant showed a gap longer than 1 hour in the time of dim light melatonin onset

197 (DLMO) between the conditions (medium breaks vs. bright breaks). DLMO was

198 determined by linear interpolation between two time points at which melatonin

199 concentration crossed the $3.0 \mathrm{pg} / \mathrm{ml}$ threshold (Benloucif et al. 2008).

\section{Statistical analysis}

202 In statistical comparisons between the conditions (medium break vs. bright break or

203 medium break vs. dim break) for the melatonin profile, subjective sleepiness (KSS),

204 performance (PVT), repeated-measures two-way ANOVA (SPSS 23.0, IBM $^{\odot}$ SPSS $^{\odot}$ 
205 Statistics) with light conditions and time (during the simulated night work) as 206 independent factors was conducted. Greenhouse-Geisser correction was performed 207 when Mauchily's sphericity assumption was largely violated. A two-sided, paired 208 Student's t-test was used for planned comparisons between the light conditions during 209 the night work when a significant interaction between the independent factors was 210 found. For the comparison of numbers of PVT lapses between the conditions, the 211 Wilcoxon signed-rank test was conducted. A P-value of less than 0.05 was considered 212 statistically significant.

213

214 Results

\section{Melatonin suppression}

216 Figure 2 shows the melatonin profiles obtained from each experiment. In both

217 experiments, melatonin gradually increased under the dim light condition (21:00-

218 01:00h) but was immediately attenuated by light exposure (550 lx) from 01:00h in both

219 experiments. However, the aspects of melatonin suppression were different with the 220 light conditions during breaks.

In Experiment 1 (Figure 2a), repeated-measures two-way ANOVA with light condition (medium break vs. dim break) and time (01:00 06:00h) showed a main effect in light condition $\left(\mathrm{F}_{1,11}=6.966, P=0.027\right)$ but not in time $\left(\mathrm{F}_{1.951,21.463}=0.511, P=\right.$ $0.603, \mathrm{~ns})$. There was a significant interaction between condition and time $\left(\mathrm{F}_{2.827,31.095}=\right.$ $3.826, P=0.021)$. A paired t-test for melatonin concentrations at each time point showed that the dim break resulted in greater melatonin suppression than did the medium break at 04:00,05:00, and 06:00h $(P=0.011, P=0.007$, and $P=0.001$, respectively). A comparison of the melatonin AUCs between the conditions showed that there was a significant tendency for lower melatonin concentration in the dim break condition compared to that in the medium break condition.

Similarly, in Experiment 2 (Figure 2b), repeated-measures two-way ANOVA with light condition (medium break vs. bright break) and time (01:00 06:00h) showed a main effect in light condition $\left(\mathrm{F}_{1,8}=9.837, P=0.014\right)$ but not in time $\left(\mathrm{F}_{5,40}=0.981, P\right.$ $=0.441, \mathrm{~ns})$. A significant interaction between condition and time was found $\left(\mathrm{F}_{5,40}=\right.$ 4.484, $P=0.002$ ). A paired t-test for melatonin concentrations at each time point showed that the bright break resulted in lower melatonin suppression than did the medium break at 04:00, 05:00, and 06:00h $(P=0.009, P=0.038$, and $\mathrm{P}=0.001$,

238 respectively). There was also a significant tendency for higher melatonin concentration 
239 in the bright breaks than in the medium breaks at 05:00h (paired t-test, $P=0.050$ ). The

240 melatonin AUC in the bright breaks was significantly greater than that in the medium

241 breaks.

242

\section{Subjective sleepiness (KSS)}

244 The results for subjective sleepiness are shown in Figure 3. In Experiment 1 (dim

245 breaks), subjective sleepiness gradually increased over time during the simulated night

246 work span $\left(\mathrm{F}_{8,34.031}=9.066 ; p<0.001\right)$, but it showed almost the same pattern in the

247 conditions (medium vs. $\operatorname{dim})\left(\mathrm{F}_{1,11}=0.723 ; p=0.413\right)$. In addition, no significant

248 interaction was found between conditions and time $\left(\mathrm{F}_{8,42.824}=1.709 ; p=0.167\right)$.

249 Similarly, in Experiment 2 (bright breaks), there was a significant main effect of time

$250\left(\mathrm{~F}_{8,64}=14.030 ; p<0.001\right)$, but no main effect of condition $\left(\mathrm{F}_{1,8}=0.018 ; p=0.897\right)$,

251 and no interaction between condition and time $\left(\mathrm{F}_{8,64}=1.195 ; p=0.316\right)$ were found.

252

$253 \quad \boldsymbol{P V T}$

254 Figure 4 shows the results for reaction speed (mean reciprocal reaction time: mean

255 1/RT) (Basner \& Dinges 2011) in the two experiments. In ANOVA analysis for mean

$2561 / \mathrm{RT}$, there were significant main effects of time in Experiment 1 (dim breaks) $\left(\mathrm{F}_{8,27.005}\right.$

$257=8.729 ; p=0.001)$ and in Experiment 2 (bright breaks) $\left(\mathrm{F}_{8,16.421}=11.593 ; p=0.001\right)$.

258 However, mean 1/RT was not significantly different between conditions in both

259 experiments $\left(\mathrm{F}_{1,11}=0.0004 ; p=0.984\right.$ in Experiment $1, \mathrm{~F}_{1,8}=0.018 ; p=0.897$ in

260 Experiment 2). Also, no interactions between conditions and time were found $\left(\mathrm{F}_{8,88}=\right.$

$2610.927 ; p=0.499$ in Experiment $1, \mathrm{~F}_{8,22.129}=1.073 ; p=0.377$ in Experiment 2).

262 A comparison of the numbers of lapses at each time point (Wilcoxon signed-

263 rank test) showed that there was no significant difference at any time points between

264 medium breaks and dim breaks and between medium breaks and bright breaks (Figure

$2654)$.

266

267 Discussion

268 In the present study, we investigated whether periodic short-duration exposures (for 10

$269 \mathrm{~min}$ at hourly intervals) to very bright (bright breaks) or very dim light (dim breaks)

270 affect physiological responses including melatonin suppression, subjective sleepiness,

271 and performance during exposure to constant medium intensity light. We found that both the

272 dim breaks and bright breaks indirectly, rather than directly, affected melatonin suppression 
273 during the experiments: the dim breaks promoted melatonin suppression, whereas the 274 bright breaks diminished melatonin suppression. A possible reason for these results is 275 that the dim breaks or the bright breaks sensitized or desensitized pineal melatonin to the subsequent constant light exposure during the experiment.

The effect of brief light exposure during each break on melatonin synthesis was first observed at the 04:00h time point, just before the 3rd break in both experiments, and the sensitized or desensitized states lasted until the end of the experiment (i.e.,

280 06:00h). However, this does not mean that the first break had no effect at all, but rather it seems that adaptation to dim light for 4 hours before the start of the experiment caused strong melatonin suppression by sensitizing melatonin responsiveness to light (see melatonin suppressions at 02:00h in Figure 2). This may indicate that melatonin sensitivity can be changed shortly after short-duration exposure to light. Our results might suggest that using brief photo-adaptation probably enables real-time adjustment of melatonin sensitivity against a current light stimulus depending on relative photic intensity.

Although the results of the present study are similar to previous findings of prior light history having an effect on melatonin (see Introduction section), the underlying mechanisms responsible for the results appear to be different. In previous studies, it is more likely to be a compensatory adaptation by long-term adaptation of photoreceptors to a given photo-environment. On the other hand, although the mechanisms by which short adaptations during breaks contribute to melatonin sensitivity are not known, they seem to be associated with bleaching and recovery mechanism of photoreceptors in the retina. For instance, the bleached photopigments might be partially recovered by the dim breaks, leading to an increase in gain of the phototransduction cascade. Likewise, profound bleaching of a substantial fraction of the photopigments due to exposure to very bright light during the bright break might lead to attenuation of melatonin sensitivity to the subsequent light (Fain et al. 2001). On the other hand, visual

300 photoreceptors, including rods and cones, saturate at a relatively low-intensity level of 301 light (Lucas et al. 2003). Given that mRGC compensate the functional limitations of the visual photoreceptors for higher light intensities (Gooley et al. 2012), it might be more important to understand whether the mRGC has such capacity of light adaptation. The photopigment melanopsin has been shown to be homologous to invertebrate opsin (rhabdomeric opsin) (Shichida \& Matsuyama 2009), and it has therefore been

306 hypothesized that melanopsin uses the rhabdomeric phototransduction cascade (Hillman 
307 et al. 1983). Although controversial, several previous studies have provided evidence of

308 a bi- or tri-stable signaling state in mammalian melanopsin including, for example, red-

309 light enhancement for pupil response to blue light (Graham et al. 2008; Mure et al.

310 2009; Emanuel \& Do 2015).

311 Another in vitro study demonstrated that prior light stimulus alters the sensitivity

312 of rat mRGC to subsequent light exposure in a way similar to that of photoreceptor

313 adaptation, rather than neural network adaptation: a brief flash desensitized the cells

314 whereas darkness re-sensitized the cells without synaptic inputs from rods and cones

315 (Wong et al. 2005). According to the study, mRGC completed light adaptation (i.e.,

316 desensitization) within $5 \mathrm{~min}$. On the other hand, the kinetics of dark adaptation

317 appeared to be even slower for mRGC than for rods, as the cell showed a striking

318 increase in sensitivity after 30 40 min of dark adaptation and kept increasing for at least

$3192 \mathrm{~h}$ and $40 \mathrm{~min}$. More recent studies, however, have shown that synaptic inputs from the

320 classical photoreceptors (i.e., rods and cones) via the inner plexiform layer to mRGC

321 increase the sensitivity of mRGC to light (Wong et al. 2007). Based on these results,

322 exposure to very bright light for $10 \mathrm{~min}$ (i.e., bright breaks) might be sufficient to cause

323 a massive decrease in the photosensitivity of mRGC, and this phenomenon likely lead

324 to the attenuation of melatonin suppression in Experiment 1. However, recovery in near

325 darkness for $10 \mathrm{~min}$ (i.e., dim breaks) was probably not sufficient to elicit a significant

326 increase in the photosensitivity of $\mathrm{mRGC}$; rather, synaptically mediated signals from

327 partially dark-adapted classical photoreceptors might be more responsible for the

328 promotion of melatonin suppression in Experiment 2.

There were no remarkable effects of dim breaks or bright breaks on alertness,

330 i.e., subjective sleepiness and performance of the psychomotor vigilance task. As an

331 indirect alerting effect of light via retinal projection to the SCN, the magnitude of

332 melatonin suppression was thought to be involved in subjective sleepiness (Cajochen

333 2007). Nonetheless, subjective sleepiness or reaction speed (i.e., mean 1/RT) was

334 consistently increased or decreased over time in a similar pattern regardless of the break

335 conditions. Similarly, unlike medium breaks, bright breaks and dim breaks did not have

336 an additional effect on the number of lapses. On the other hand, as a direct alerting

337 effect of light (Souman et al. 2018), the bright breaks were expected to be able to delay

338 the decrease in alertness. However, we could not find such a beneficial effect even

339 when we compared the reaction velocities or numbers of lapses before and after the

340 bright breaks (Figure $4 \mathrm{~b}$ and Figure $5 \mathrm{~b}$ ). Moreover, although acute reduction of 
341 subjective sleepiness tended to emerge during each break session, it seems to be a 342 temporal effect associated with moving to a break room or a light stretch during each 343 break session, rather than than a direct alerting effect of light during each break.

344 Nevertheless, it should be noted that the participants were continuously exposed to 345 medium intensity light during the simulated night work, and this might have diluted the 346 additional effects of bright breaks or dim breaks on alertness considering the dose-response 347 relationship between light intensity and alertness (Cajochen et al. 2000). Also, high 348 sleep pressure due to prior wakefulness might be partially responsible for the results, since the participants did not take a nap before the start of the experiments.

Epidemiological studies conducted over the past few decades have suggested adverse relationships of night shift work with acute and chronic adverse health problems (Kantermann et al. 2010; Parent et al. 2012; Evans \& Davidson 2013; Kamdar et al. 2013). Direct effects of exposure to bright white light, especially blue-enriched light, on the circadian system, such as melatonin suppression and circadian misalignment, for example, between the biological clock and the social-behavioral cycle have been suspected as factors involved in the risks (Wittmann et al. 2006; Touitou et al. 2017). Nonetheless, in some ways, exposure to bright white light (i.e., blue-enriched light) is also helpful for keeping night workers awake and providing better visibility, leading to better performance and fewer accidents due to human errors (Cajochen 2007; Chellappa et al. 2011; Kraneburg et al. 2017). For attenuation of melatonin suppression without a negative effect on performance, the use of lighting with less short-wavelength components (Kozaki et al. 2008) and wearing blue light-filtering goggles (Kayumov et al. 2005), or a red-visor cap (Higuchi et al. 2011), have been proposed. In addition to these proposals, a countermeasure for night shift workers is also suggested by our findings that bright breaks can reduce melatonin suppression by light without having adverse effects on sleepiness or performance.

However, a field study in which the effect of bright light exposure during a short break ( $\sim 20 \mathrm{~min})$ in night work on melatonin was investigated showed no such desensitization in melatonin suppression. In that field study, the subjects showed greater melatonin suppression in night work when they took a break with exposure to bright light $(2500 \mathrm{~lx})$ than when they took a break with exposure to normal light (300 1x)

372 (Lowden et al. 2004). However, a limitation of that field study is that the timing and 373 duration of the breaks were not strictly controlled. Another limitation is that the subjects 374 were allowed to leave the workplace for a short period. These limitations, however, 
375 rather remind us about a question if similar results could be obtained by conducting 376 bright breaks in a real night workplace. Additionally, it is uncertain in the present study 377 whether the hourly repetitive execution of breaks was essential to achieve persistent 378 effects on melatonin suppression. Although taking rest breaks is known to be effective 379 for decreasing accident risks, recovering from physical fatigue and maintaining arousal 380 level, taking breaks more than once per hour tends to disturb work (Tucker 2003). In 381 this regard, it is essential to clarify the minimum number of breaks that is necessary to 382 obtain the same results as those in the present study.

383 In the present study, although the pace of melatonin synthesis was remarkably 384 diminished after light exposure ( $5501 \mathrm{~lx})$, we did not observe dramatic melatonin 385 suppression as found in some previous studies using a protocol and illuminance level 386 similar to the present study. For example, McIntyre et al. (1989) reported that 1-h light 387 exposure (500 lx) from midnight caused about 40\% suppression of melatonin compared 388 to the melatonin concentration just before light exposure (Mcintyre et al. 1989). Laakso 389 et al. (1993) reported that melatonin suppression following 1-h light exposure from 390 23:00h amounted to as much as 53\% (Laakso et al. 1993). Ethnicity might be partially 391 responsible for the inconsistency in melatonin suppression induced by nocturnal light 392 exposure. Higuchi et al. (2007) reported that melatonin suppression following 2-h light 393 exposure (1000 1x) was greater in Caucasian than Asian subjects (Higuchi et al. 2007a). 394 Although Aoki et al. (1998) also found that melatonin suppression amounted to as much 395 as 40.1\% following 2-h light exposure (500 lx) in Asian subjects (Aoki et al. 1998), 396 dark adaptation by 5-h sleep before the light exposure possibly influenced the result 397 (Jasser et al. 2006). Subjects in the previous study were directly exposed to a specially 398 designed light source in a fixed position. However, in the present study, we used ceiling 399 light, and the gaze of each participant was not strictly fixed; hence, the light intensity 400 reaching the retina might have been less than $550 \mathrm{~lx}$ in the present study.

401 There is a question that remains unanswered: Can bright breaks or dim breaks 402 modify the circadian phase shift caused by light exposure? There have been practical 403 interventions using intermittent light to entrain the circadian clock of shift workers to 404 long-term night shift duty (Baehr et al. 1999; Crowley et al. 2003; Smith et al. 2009; 405 Smith \& Eastman 2012). Intermittent light was used for multiple days in those previous 406 studies, mainly to delay the phase of the circadian pacemaker. However, the results for 407 melatonin in the present study indicate the possibility that circadian phase delay during 408 night work can not only be promoted by conducting dim breaks, but it can also be 
attenuated by conducting bright breaks. Given the greater melatonin suppression in the condition of dim breaks than in the condition of medium breaks, conducting dim breaks during night work can probably cause a larger phase delay than can continuous exposure to medium intensity light. Modulation of circadian phase to both advance and delay might be easier by conducting bright or dim breaks based on the human phase response curve (St Hilaire et al. 2012). This study has several limitations. We measured salivary melatonin levels at hourly intervals; hence, acute effects of breaks on melatonin could not be determined in the present study. All of the participants in this study were healthy young male adults. However, inter-individual differences in the sensitivity of pineal melatonin have been shown in previous studies. Although there is still lack of agreement, there has been an accumulation of evidence indicating an age-dependent difference in pineal melatonin sensitivity (Charman 2003; Higuchi et al. 2014; Lee et al. 2018). Also, one study has suggested greater sensitivity in females than in males (Monteleone et al. 1995). The experiments in this study were conducted in different seasons: The experiment for dim breaks was conducted in summer (July), while the experiment for bright breaks was conducted in winter (from January to February). It has been reported that melatonin suppression by light at night is greater in winter than in summer (Higuchi et al. 2007b). Therefore, it is necessary to verify the reproducibility of our findings for different seasons.

In addition, inter-individual differences in the photo-sensitivity of pineal melatonin have been shown in previous studies (Higuchi et al. 2008; Santhi et al. 2012; Phillips et al. 2019). Indeed, in the present study, some participants showed strong melatonin suppression during light exposure, while others, especially participants who had a relatively low melatonin level at 01:00h (e.g., below $10 \mathrm{pg} / \mathrm{ml}$ ), showed weak melatonin suppression. Furthermore, some participants showed quick recovery from the melatonin suppression and an increase in melatonin concentration over time. It remains unclear what causes the individual differences, but several recent studies have suggested that genetic variations in the clock genes are associated with inter-individual differences in melatonin suppression (Chellappa et al. 2012; Akiyama et al. 2017). Further investigation should be carried out to identify the individual differences in nonvisual photo-sensitivity.

Our findings suggest that periodic exposure to light for a short duration during exposure to constant light affects melatonin sensitivity to the constant light depending 
443 on the difference between light intensities in the light conditions (i.e., exposure to short

444 light vs. exposure to constant light). In most previous studies, the effects of light with

445 fixed intensity and/or spectral composition on the circadian system were investigated.

446 However, humans generally do not stay at the same place for long duration; the light

447 environment surrounding us frequently changes in real life. In this regard, the findings

448 in the present study suggest that exposure to light of various intensities at night could be

449 a factor influencing the light-induced melatonin suppression in real life.

450

\section{Acknowledgments}

452 We thank Takuya Tsuyama at Kyushu University for his help in the preparation of this

453 study. This work was supported by the Japan Society for the Promotion of Science

454 under Grant-in-Aid for Scientific Research (KAKENHI) number 19H03316.

455

456 Declaration of interest statement

457 The authors report no conflict of interest. 


\section{References}

Akiyama T, Katsumura T, Nakagome S, Lee SI, Joh K, Soejima H, Fujimoto K, Kimura R, Ishida H, Hanihara T, Yasukouchi A, Satta Y, Higuchi S, Oota H. 2017. An ancestral haplotype of the human PERIOD2 gene associates with reduced sensitivity to light-induced melatonin suppression. Plos One. 12.

Aoki H, Yamada N, Ozeki Y, Yamane H, Kato N. 1998. Minimum light intensity required to suppress nocturnal melatonin concentration in human saliva. Neurosci Lett. 252:91-94.

Baehr EK, Fogg LF, Eastman CI. 1999. Intermittent bright light and exercise to entrain human circadian rhythms to night work. Am J Physiol-Reg I. 277:R1598R1604.

Basner M, Dinges DF. 2011. Maximizing Sensitivity of the Psychomotor Vigilance Test (PVT) to Sleep Loss. Sleep. 34:581-591.

Benloucif S, Burgess HJ, Klerman EB, Lewy AJ, Middleton B, Murphy PJ, Parry BL, Revell VL. 2008. Measuring Melatonin in Humans. J Clin Sleep Med. 4:66-69.

Brainard GC, Hanifin JP, Greeson JM, Byrne B, Glickman G, Gerner E, Rollag MD. 2001. Action spectrum for melatonin regulation in humans: evidence for a novel circadian photoreceptor. J Neurosci. 21:6405-6412.

Cajochen C. 2007. Alerting effects of light. Sleep Med Rev. 11:453-464.

Cajochen C, Zeitzer JM, Czeisler CA, Dijk DJ. 2000. Dose-response relationship for light intensity and ocular and electroencephalographic correlates of human alertness. Behav Brain Res. 115:75-83.

Chang AM, Santhi N, St Hilaire M, Gronfier C, Bradstreet DS, Duffy JF, Lockley SW, Kronauer RE, Czeisler CA. 2012. Human responses to bright light of different durations. J Physiol-London. 590:3103-3112.

Chang AM, Scheer FAJL, Czeisler CA. 2011. The human circadian system adapts to prior photic history. J Physiol-London. 589:1095-1102.

Charman WN. 2003. Age, lens transmittance, and the possible effects of light on melatonin suppression. Ophthalmic Physiol Opt. 23:181-187.

Chellappa SL, Steiner R, Blattner P, Oelhafen P, Gotz T, Cajochen C. 2011. Non-Visual Effects of Light on Melatonin, Alertness and Cognitive Performance: Can BlueEnriched Light Keep Us Alert? PLoS One. 6.

Chellappa SL, Viola AU, Schmidt C, Bachmann V, Gabel V, Maire M, Reichert CF, Valomon A, Gotz T, Landolt HP, Cajochen C. 2012. Human Melatonin and Alerting Response to Blue-Enriched Light Depend on a Polymorphism in the Clock Gene PER3. J Clin Endocrinol Metab. 97:E433-E437.

Crowley SJ, Lee C, Tseng CY, Fogg LF, Eastman CI. 2003. Combinations of bright light, scheduled dark, sunglasses, and melatonin to facilitate circadian entrainment to night shift work. J Biol Rhythms. 18:513-523.

Emanuel AJ, Do MTH. 2015. Melanopsin Tristability for Sustained and Broadband Phototransduction. Neuron. 85:1043-1055.

Enezi J, Revell V, Brown T, Wynne J, Schlangen L, Lucas R. 2011. A "melanopic" spectral efficiency function predicts the sensitivity of melanopsin photoreceptors to polychromatic lights. J Biol Rhythms. 26:314-323.

Evans JA, Davidson AJ. 2013. Health Consequences of Circadian Disruption in Humans and Animal Models. Prog Mol Biol Transl. 119:283-323.

Fain GL, Matthews HR, Cornwall MC, Koutalos Y. 2001. Adaptation in vertebrate photoreceptors. Physiol Rev. 81:117-151. 
507 Gimenez MC, Beersma DG, Bollen P, van der Linden ML, Gordijn MC. 2014. Effects of a chronic reduction of short-wavelength light input on melatonin and sleep patterns in humans: evidence for adaptation. Chronobiol Int. 31:690-697.

Gooley JJ, Mien IH, St Hilaire MA, Yeo SC, Chua ECP, van Reen E, Hanley CJ, Hull JT, Czeisler CA, Lockley SW. 2012. Melanopsin and Rod-Cone Photoreceptors Play Different Roles in Mediating Pupillary Light Responses during Exposure to Continuous Light in Humans. J Neurosci. 32:14242-14253.

Graham DM, Wong KY, Shapiro P, Frederick C, Pattabiraman K, Berson DM. 2008. Melanopsin ganglion cells use a membrane-associated rhabdomeric phototransduction cascade. J Neurophysiol. 99:2522-2532.

Gronfier C, Wright KP, Kronauer RE, Jewett ME, Czeisler CA. 2004. Efficacy of a single sequence of intermittent bright light pulses for delaying circadian phase in humans. Am J Physiol-Endoc M. 287:E174-E181.

Hebert M, Martin SK, Lee C, Eastman CI. 2002. The effects of prior light history on the suppression of melatonin by light in humans. J Pineal Res. 33:198-203.

Higuchi S, Fukuda T, Kozaki T, Takahashi M, Miura N. 2011. Effectiveness of a Redvisor Cap for Preventing Light-induced Melatonin Suppression during Simulated Night Work. J Physiol Anthropol. 30:251-258.

Higuchi S, Ishibashi K, Aritake S, Enomoto M, Hida A, Tamura M, Kozaki T, Motohashi Y, Mishima K. 2008. Inter-individual difference in pupil size correlates to suppression of melatonin by exposure to light. Neurosci Lett. 440:23-26.

Higuchi S, Motohashi Y, Ishibashi K, Maeda T. 2007a. Influence of eye colors of Caucasians and Asians on suppression of melatonin secretion by light. Am J Physiol-Reg I. 292:R2352-R2356.

Higuchi S, Motohashi Y, Ishibashi K, Maeda T. 2007b. Less exposure to daily ambient light in winter increases sensitivity of melatonin to light suppression. Chronobiol Int. 24:31-43.

Higuchi S, Nagafuchi Y, Lee S, Harada T. 2014. Influence of Light at Night on Melatonin Suppression in Children. J Clin Endocrinol Metab. 99:3298-3303.

Hillman P, Hochstein S, Minke B. 1983. Transduction in Invertebrate Photoreceptors Role of Pigment Bistability. Physiol Rev. 63:668-772.

Ishihara K, Saitoh T, Inoue Y, Miyata Y. 1984. Validity of the Japanese Version of the Morningness-Eveningness Questionnaire. Percept Mot Skills. 59:863-866.

Jasser SA, Hanifin JP, Rollag MD, Brainard GC. 2006. Dim light adaptation attenuates acute melatonin suppression in humans. J Biol Rhythms. 21:394-404.

Kamdar BB, Tergas AI, Mateen FJ, Bhayani NH, Oh J. 2013. Night-shift work and risk of breast cancer: a systematic review and meta-analysis. Breast Cancer Res Treat. 138:291-301.

Kantermann T, Juda M, Vetter C, Roenneberg T. 2010. Shift-work research: Where do we stand, where should we go? Sleep and Biological Rhythms. 8:95-105.

Kayumov L, Casper RF, Hawa RJ, Perelman B, Chung SA, Sokalsky S, Shapiro CM. 2005. Blocking low-wavelength light prevents nocturnal melatonin suppression with no adverse effect on performance during simulated shift work. J Clin Endocrinol Metab. 90:2755-2761.

Kozaki T, Koga S, Toda N, Noguchi H, Yasukouchi A. 2008. Effects of short wavelength control in polychromatic light sources on nocturnal melatonin secretion. Neurosci Lett. 439:256-259. 
Kraneburg A, Franke S, Methling R, Griefahn B. 2017. Effect of color temperature on melatonin production for illumination of working environments. Appl Ergon. 58:446-453.

Kronauer RE, Forger DB, Jewett ME. 1999. Quantifying human circadian pacemaker response to brief, extended, and repeated light stimuli over the phototopic range. J Biol Rhythms. 14:500-515.

Laakso ML, Hatonen T, Stenberg D, Alila A, Smith S. 1993. One-Hour Exposure to Moderate Illuminance (500-Lux) Shifts the Human Melatonin Rhythm. J Pineal Res. 15:21-26.

Lamb TD, Pugh EN. 2004. Dark adaptation and the retinoid cycle of vision. Prog Retin Eye Res. 23:307-380.

Lee SI, Matsumori K, Nishimura K, Nishimura Y, Ikeda Y, Eto T, Higuchi S. 2018. Melatonin suppression and sleepiness in children exposed to blue-enriched white LED lighting at night. Physiol Rep. 6.

Lowden A, Akerstedt T, Wibom R. 2004. Suppression of sleepiness and melatonin by bright light exposure during breaks in night work. J Sleep Res. 13:37-43.

Lucas RJ, Hattar S, Takao M, Berson DM, Foster RG, Yau KW. 2003. Diminished pupillary light reflex at high irradiances in melanopsin-knockout mice. Science. 299:245-247.

Lunn RM, Blask DE, Coogan AN, Figueiro M, Gorman MR, Hall JE, Hansen J, Nelson RJ, Panda S, Smolensky MH, Stevens RG, Turek FW, Vermeulen R, Carreon T, Caruso CC, Lawson CC, Thayer KA, Twery MJ, Ewens AD, Garner SC, Schwingl PJ, Boyd WA. 2017. Health consequences of electric lighting practices in the modern world: A report on the National Toxicology Program's workshop on shift work at night, artificial light at night, and circadian disruption. Sci Total Environ. 607:1073-1084.

Macchi MM, Bruce JN. 2004. Human pineal physiology and functional significance of melatonin. Front Neuroendocrinol. 25:177-195.

Mcintyre IM, Norman TR, Burrows GD, Armstrong SM. 1989. Human Melatonin Suppression by Light Is Intensity Dependent. J Pineal Res. 6:149-156.

Monteleone P, Esposito G, Larocca A, Maj M. 1995. Does Bright Light Suppress Nocturnal Melatonin Secretion More in Women Than Men. J Neural TransmGen. 102:75-80.

Mure LS, Cornut PL, Rieux C, Drouyer E, Denis P, Gronfier C, Cooper HM. 2009. Melanopsin Bistability: A Fly's Eye Technology in the Human Retina. PLoS One. 4.

Parent ME, El-Zein M, Rousseau MC, Pintos J, Siemiatycki J. 2012. Night Work and the Risk of Cancer Among Men. Am J Epidemiol. 176:751-759.

Phillips AJK, Vidafar P, Burns AC, McGlashan EM, Anderson C, Rajaratnam SMW, Lockley SW, Cain SW. 2019. High sensitivity and interindividual variability in the response of the human circadian system to evening light. Proc Natl Acad Sci U S A. 116:12019-12024.

Rahman SA, Hilaire MAS, Chang AM, Santhi N, Duffy JF, Kronauer RE, Czeisler CA, Lockley SW, Klerman EB. 2017. Circadian phase resetting by a single shortduration light exposure. Jci Insight. 2.

Rimmer DW, Boivin DB, Shanahan TL, Kronauer RE, Duffy JF, Czeisler CA. 2000. Dynamic resetting of the human circadian pacemaker by intermittent bright light. Am J Physiol-Reg I. 279:R1574-R1579.

Santhi N, Thorne HC, van der Veen DR, Johnsen S, Mills SL, Hommes V, Schlangen LJM, Archer SN, Dijk DJ. 2012. The spectral composition of evening light and 
605

606

607

608

609

610

611

612

613

614

615

616

617

618

619

620

621

622

623

624

625

626

627

628

629

630

631

632

633

634

635

636

637

638

639

640

641

642

643

644

645 individual differences in the suppression of melatonin and delay of sleep in humans. J Pineal Res. 53:47-59.

Shichida Y, Matsuyama T. 2009. Evolution of opsins and phototransduction. Philos T R Soc B. 364:2881-2895.

Smith KA, Schoen MW, Czeisler CA. 2004. Adaptation of human pineal melatonin suppression by recent photic history. J Clin Endocrinol Metab. 89:3610-3614.

Smith MR, Eastman CI. 2012. Shift work: health, performance and safety problems, traditional countermeasures, and innovative management strategies to reduce circadian misalignment. Nat Sci Sleep. 4:111-132.

Smith MR, Fogg LF, Eastman CI. 2009. Practical Interventions to Promote Circadian Adaptation to Permanent Night Shift Work: Study 4. J Biol Rhythms. 24:161172.

Smolensky MH, Hermida RC, Reinberg A, Sackett-Lundeen L, Portaluppi F. 2016. Circadian disruption: New clinical perspective of disease pathology and basis for chronotherapeutic intervention. Chronobiol Int. 33:1101-1119.

Smolensky MH, Sackett-Lundeen LL, Portaluppi F. 2015. Nocturnal light pollution and underexposure to daytime sunlight: Complementary mechanisms of circadian disruption and related diseases. Chronobiol Int. 32:1029-1048.

Souman JL, Tinga AM, te Pas SF, van Ee R, Vlaskamp BNS. 2018. Acute alerting effects of light: A systematic literature review. Behav Brain Res. 337:228-239.

St Hilaire MA, Gooley JJ, Khalsa SBS, Kronauer RE, Czeisler CA, Lockley SW. 2012. Human phase response curve to a $1 \mathrm{~h}$ pulse of bright white light. J PhysiolLondon. 590:3035-3045.

Touitou Y, Reinberg A, Touitou D. 2017. Association between light at night, melatonin secretion, sleep deprivation, and the internal clock: Health impacts and mechanisms of circadian disruption. Life Sci. 173:94-106.

Tucker P. 2003. The impact of rest breaks upon accident risk, fatigue and performance: a review. Work Stress. 17:123-137.

Weaver DR. 1998. The suprachiasmatic nucleus: A 25-year retrospective. J Biol Rhythms. 13:100-112.

Wittmann M, Dinich J, Merrow M, Roenneberg T. 2006. Social jetlag: Misalignment of biological and social time. Chronobiol Int. 23:497-509.

Wong KY, Dunn FA, Berson DM. 2005. Photoreceptor adaptation in intrinsically photosensitive retinal ganglion cells. Neuron. 48:1001-1010.

Wong KY, Dunn FA, Graham DM, Berson DM. 2007. Synaptic influences on rat ganglion-cell photoreceptors. J Physiol-London. 582:279-296.

Zeitzer JM, Dijk DJ, Kronauer RE, Brown EN, Czeisler CA. 2000. Sensitivity of the human circadian pacemaker to nocturnal light: melatonin phase resetting and suppression. J Physiol-London. 526:695-702. 
646 Table 1. Light conditions used in this study

\begin{tabular}{lcccc}
\hline & $\begin{array}{c}\text { Night Shift Light } \\
\text { (01:00-06:00h) }\end{array}$ & \multicolumn{3}{c}{$\begin{array}{c}10 \text { Min Breaks } \\
\text { (4 per night, 1/h) }\end{array}$} \\
\cline { 2 - 5 } & $\begin{array}{c}\text { Medium } \\
\text { Intensity Light }\end{array}$ & $\begin{array}{c}\text { Medium } \\
\text { Intensity Light }\end{array}$ & Bright Light & Dim Light \\
\hline Illuminance (Ix) & 550 & 430 & 4700 & 1 \\
Color temperature (K) & 4500 & 3850 & 5000 & - \\
Photon flux ( $\left.\log _{10} 1 / \mathrm{cm}^{2} / \mathrm{sec}\right)$ & 14.70 & 14.54 & 15.62 & 12.63 \\
Melanopic lux & 83.37 & 52.86 & 787.84 & 0.09 \\
\hline
\end{tabular}

647 
648 Figure 1. Experimental protocol (a) and details of the experimental tasks during the

649 break and during the simulated night work (b). Participants always stayed in chamber 1

650 from 13:00h to the end of the experiment (i.e., 06:00h the next morning) except for

651 when they took breaks in chamber 2.

652

653 Figure 2. Melatonin profiles (means \pm standard error) and AUCs (means + standard

654 error) in Experiment 1 (a: medium breaks vs. dim breaks) and Experiment 2 (b: medium

655 breaks vs. bright breaks). The black arrows indicate the times when breaks were

656 conducted. **: $p<0.01, *: p<0.05$

657

658 Figure 3. Subjective sleepiness (means \pm standard error) in Experiment 1 (a: medium

659 breaks vs. dim breaks) and Experiment 2 (b: medium breaks vs. bright breaks). The

660 black arrows indicate the times when breaks were conducted.

661

662 Figure 4. Cognitive performance (i.e., reaction speed [mean 1/RT]; a, b) and number of

663 lapses (c, d) in Experiment 1 (medium breaks vs. dim breaks; left columns) and

664 Experiment 2 (medium breaks vs. bright breaks; right columns). The black arrows

665 indicate the times when breaks were conducted. 
Figure 1

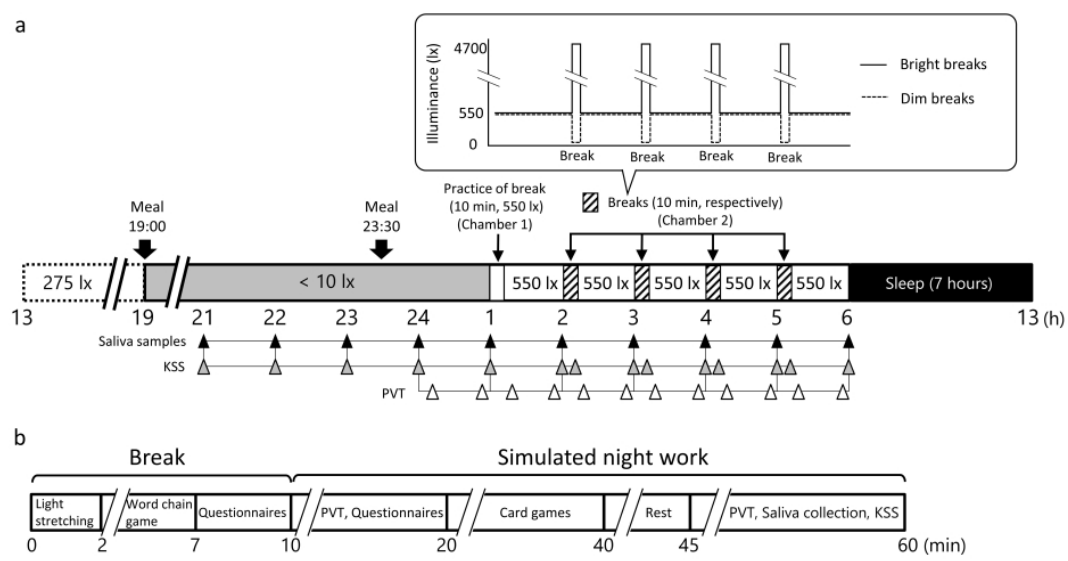

Figure 1. Experimental protocol (a) and details of the experimental tasks during the break and during the simulated night work (b). Participants always stayed in chamber 1 from 13:00h to the end of the experiment (i.e., 06:00h the next morning) except for when they took breaks in chamber 2.

$338 \times 190 \mathrm{~mm}(600 \times 600 \mathrm{DPI})$ 
Figure 2
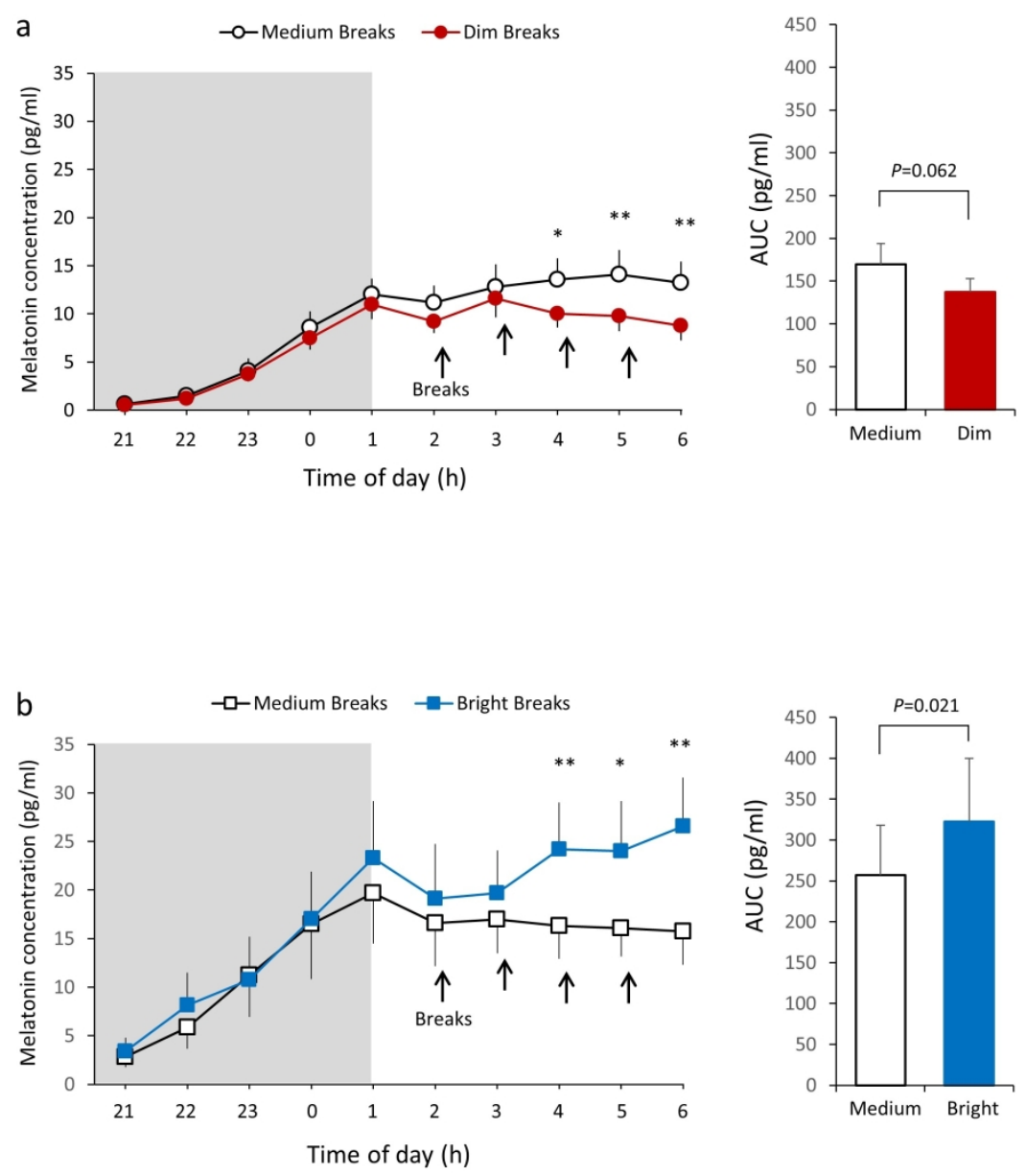

Figure 2. Melatonin profiles (means \pm standard error) and AUCs (means + standard error) in Experiment 1 (a: medium breaks vs. dim breaks) and Experiment 2 (b: medium breaks vs. bright breaks). The black arrows indicate the times when breaks were conducted. $* *: p<0.01, *: p<0.05$

$190 \times 275 \mathrm{~mm}(300 \times 300 \mathrm{DPI})$ 
Figure 3

a

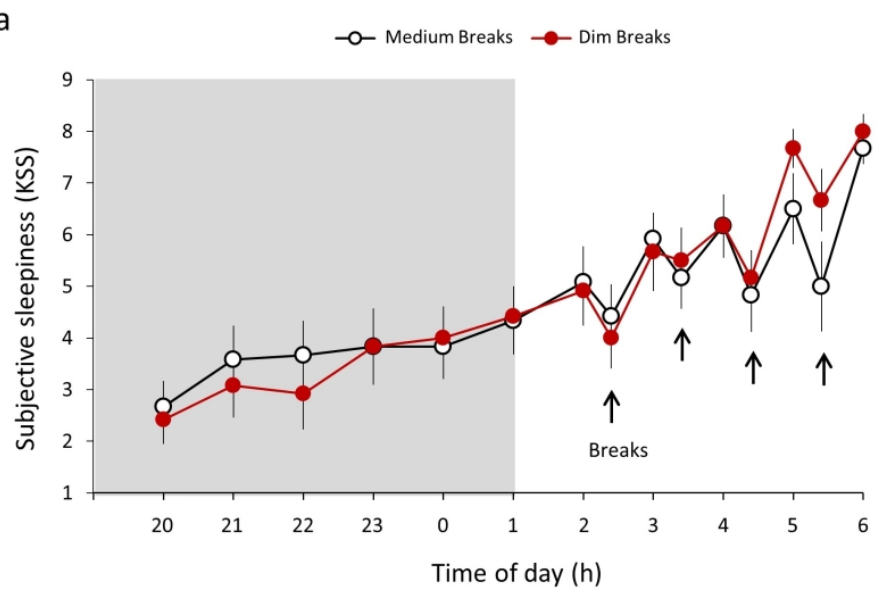

b $\neg$ - Medium Breaks $\rightarrow-$ Bright Breaks

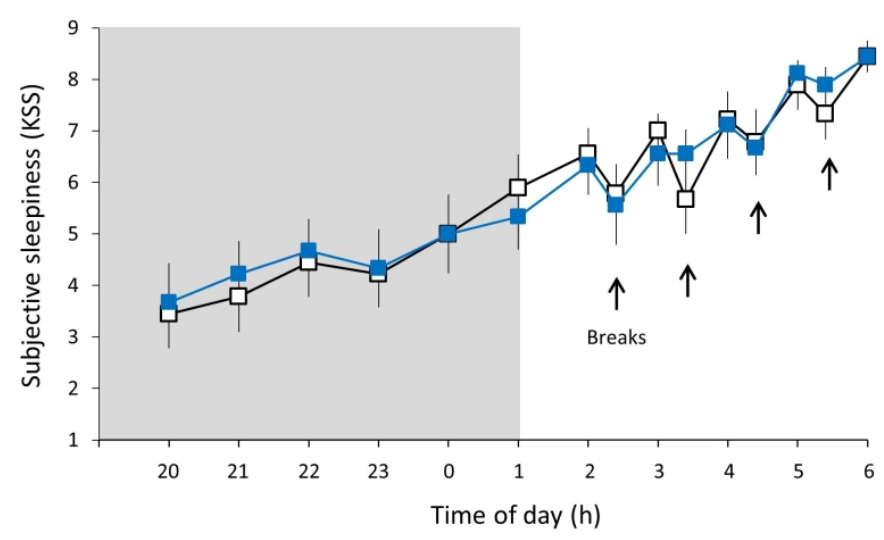

Figure 3. Subjective sleepiness (means \pm standard error) in Experiment 1 (a: medium breaks vs. dim breaks) and Experiment 2 (b: medium breaks vs. bright breaks). The black arrows indicate the times when breaks were conducted.

$190 \times 275 \mathrm{~mm}(300 \times 300 \mathrm{DPI})$ 
Figure 4
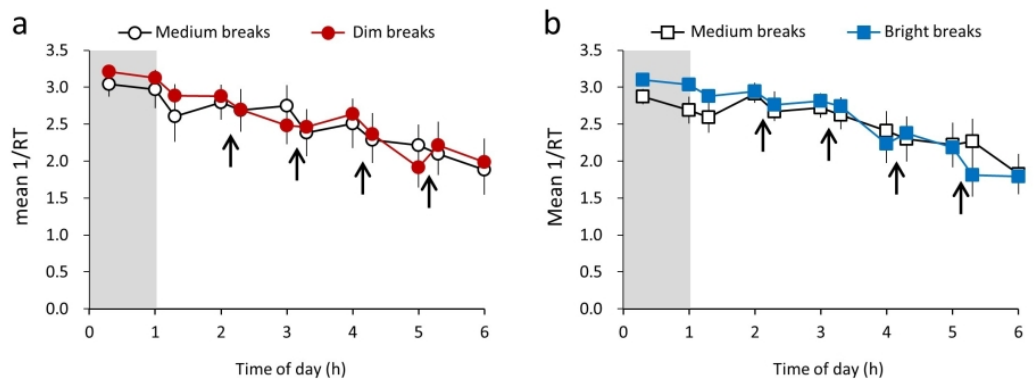

C

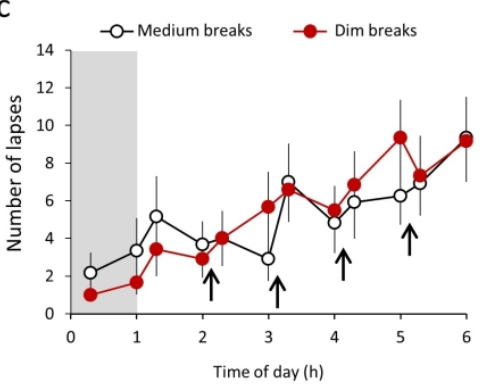

d

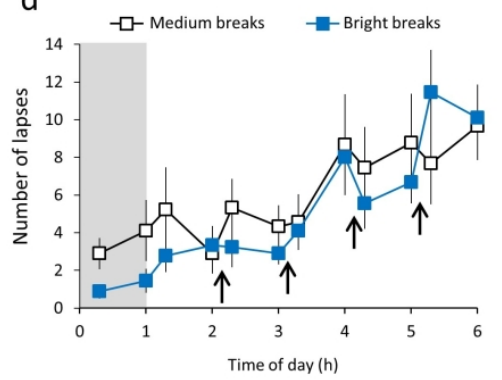

Figure 4. Cognitive performance (i.e., reaction speed [mean 1/RT]; $a, b$ ) and number of lapses (c, d) in Experiment 1 (medium breaks vs. dim breaks; left columns) and Experiment 2 (medium breaks vs. bright breaks; right columns). The black arrows indicate the times when breaks were conducted.

$190 \times 275 \mathrm{~mm}(300 \times 300 \mathrm{DPI})$ 